\title{
Predictive Analysis of Economic Chaotic Time Series Based on Chaotic Genetics Combined with Fuzzy Decision Algorithm
}

\author{
Xiuge Tan (iD) \\ School of Business, Xi'an University of Finance and Economics, Xi'an, Shaanxi 710100, China \\ Correspondence should be addressed to Xiuge Tan; 2016010022@xaufe.edu.cn
}

Received 24 January 2021; Revised 22 March 2021; Accepted 8 April 2021; Published 17 April 2021

Academic Editor: Wei Wang

Copyright (C) 2021 Xiuge Tan. This is an open access article distributed under the Creative Commons Attribution License, which permits unrestricted use, distribution, and reproduction in any medium, provided the original work is properly cited.

\begin{abstract}
The irreversibility in time, the multicausality on lines, and the uncertainty of feedbacks make economic systems and the predictions of economic chaotic time series possess the characteristics of high dimensionalities, multiconstraints, and complex nonlinearities. Based on genetic algorithm and fuzzy rules, the chaotic genetics combined with fuzzy decision-making can use simple, fast, and flexible means to complete the goals of automation and intelligence that are difficult to traditional predicting algorithms. Moreover, the new combined method's ergodicity can perform nonrepetitive searches in a global scope, hence improving the algorithm's accuracy and efficiency. On the basis of summarizing and analyzing previous research works, this paper expounded the research status and significance of the prediction of economic chaotic time series, elaborated the development background, current status, and future challenges of the combined algorithm of chaotic genetics with fuzzy decision, introduced the basic principles of chaotic genetic algorithm and fuzzy decision algorithm, constructed a prediction model for economic chaotic time series, performed parameter synchronization optimization and moderate function construction, analyzed the prediction processes of economic chaotic time series, conducted phase space reconstruction and correlation dimension calculation, and finally carried out a simulation experiment with its result analysis. The study results show that the algorithm of chaotic genetics combined with fuzzy decision-making can dynamically adjust chaotic mutation operators and summarize fussy expert experiences. The phase space of its reconstructed chaotic attractor has high-precision predictability and can find orderly processes from changeable economic results, which in turn can be used to analyze and predict the complex economic chaotic time series. The study results of this paper provide a reference for further research on predictive analysis of economic chaotic time series based on chaotic genetics combined with fuzzy decision algorithm.
\end{abstract}

\section{Introduction}

With the rapid development of chaos science, chaotic time series analysis of economic and financial system behavior has become a hot topic in the field of relevant research, and the chaotic economics developed from this field has greatly enhanced the ability of economic theory to describe reality [1]. Economic chaotic time series is a kind of irregular motion occurring in a certain economic system, which is often expressed as a discrete state, and it also is a model-generated time series with chaotic characteristics, whose entirely systemic dynamic behavior and chaotic series are predicted in the chaotic dynamic system [2]. The irreversibility in time, the multicausality online, and the uncertainty of feedbacks make the economic system itself have very complex nonlinearity; therefore, the economic chaotic time series prediction has the characteristics of high dimensionality, nonlinearity, and multiconstraints and thereby has difficulty in finding optimal solution in theory [3]. However, complexity theories such as fractal and chaos can quantify those chaotic systems and can find orderly processes from the complex and changeable economic results, which in turn can analyze and predict the complex and changeable results of economic chaotic time series. Therefore, this paper discusses the application of a new algorithm to predict and analyze economic chaotic time series in order to achieve initial expectations, obtain corresponding knowledge, and achieve desired goals [4].

There have been many published works proposing different algorithms to conduct the predictive analysis of economic chaotic time series, but they seem to have their 
downsides. Although the cognitive map method can use the optimized model to predict the future value when there are new data pairs, it fails to enable the number of blocks and units in each block unit to be expanded according to a certain growing pattern [5]. The online learning method can be linked to the correlation criterion, but this method may contain strong jitter in the overall change trend and has a certain degree of randomness [6]. The machine learning adopts a partial matching crossover correction strategy; however, it cannot ensure that the component manufacturer can call the time to meet the downstream assembly needs [7]. The chaotic genetics combined with fuzzy decision algorithm can dynamically adjust its mutation operator according to the characteristics of genetic algorithm, and its ergodicity can perform a global search with no repetition and with reduced searching blindness and randomness, thereby improving the algorithm accuracy and efficiency. Therefore, this paper attempts to combine the advantages of heuristic genetic algorithm, chaotic optimization method, and fuzzy decision method: firstly, it determines the economic time series in various industries by the improved priority method, determines the time combination state by heuristic genetic algorithm, and determines the crossover and mutation rate by fuzzy decision algorithm; secondly, it conducts parallel searches with genetic algorithm in the economic benefit distribution problem and at the same time performs global optimization near the best point through the ergodicity of chaos optimization, so as to avoid genetic algorithm falling into the local optimum and effectively improve the convergence speed [8].

On the basis of summarizing and analyzing previous research works, this paper expounded the research status and significance of economic chaotic time series prediction, elaborated the development background, current status, and future challenges of chaotic genetics combined with fuzzy decision, introduced the basic principles of chaotic genetic algorithm and fuzzy decision algorithm, constructed a prediction model for economic chaotic time series, performed parameter synchronization optimization and moderate function construction, analyzed the prediction process of economic chaotic time series, conducted phase space reconstruction and correlation dimension calculation, and finally carried out a simulation experiment and its result analysis. The study results of this paper will provide a reference for further research on predictive analysis of economic chaotic time series based on chaotic genetics combined with fuzzy decision algorithm. The detailed chapters are arranged as follows: Section 2 introduces the basic methods principles of chaotic genetic algorithm and fuzzy decision algorithm; Section 3 constructs a prediction model for the economic chaotic time series based on the algorithm of chaotic genetics combined with fuzzy decision-making; Section 4 analyzes prediction process of the economic chaotic time series based on chaotic genetics combined with fuzzy decision algorithm; Section 5 conducts a simulation experiment and performs result analysis; Section 6 is conclusion.

\section{Methods and Principles}

2.1. Chaotic Genetic Algorithm. The chaotic time series prediction can be mathematically expressed as the approximation of target $y$ with elements $x_{1}, x_{2}, \cdots, x_{n}$, and the goal of this prediction is to improve the degree of approximation. According to the knowledge of information theory, in order to increase the degree of approximate $y$ with $x_{1}, x_{2}, \cdots, x_{n}$, it is necessary to enable $y$ to obtain the maximum amount of information from the reconstructed phase space $\left[x_{1}, x_{2}, \cdots, x_{n}\right]$. From this, the following $n$-step prediction model can be formed:

$$
y_{i}=a \sum_{i=1}^{n} x_{i}+b \sum_{i=1}^{n} \frac{1}{x_{i}} .
$$

In the formula, $a$ and $b$, respectively, represent the variance of the true value and the predicted value. For costconstrained economic scheduling, the cost fitness function is used to constrain the cost of the result, so the cost fitness function for individual $x_{i}$ is defined as

$$
Q\left(x_{i}\right)=\int_{i=1}^{n} e\left[\frac{1-c\left(x_{i}-x_{i-1}\right)}{1-d\left(x_{i}+x_{i-1}\right)}\right],
$$

where $Q\left(x_{i}\right)$ is the total actual cost required by instance $x_{i} ; e$ is the budget cost of workflow; $c$ and $d$ are the center and width of the output fuzzy partition, respectively.

The fitness value is a key parameter to measure the quality of chromosomes. When the total supply and demand chain cost needs to be minimized, the fitness function can be calculated as follows:

$$
W\left(x_{i}\right)=\sum_{i=1}^{n}\left[\frac{(q-w) x_{i}}{r}-\frac{1}{x_{i}}\right],
$$

where $W\left(x_{i}\right)$ is the number of schedulable periods in each workshop; $q$ is the number of scheduling periods in supply chain; $w$ is the number of processing components; $r$ is the actual cost, that is, the value of object function.

The formation of chaotic variables requires the use of chaotic mechanisms to map the variables to be optimized to the chaotic space, and the chaos in the evolutionary process can be defined by the following equation:

$$
E_{i}=\frac{1}{n+1} \sqrt{y_{i}\left(\frac{k}{t_{i}-x_{i}}-\frac{k+1}{u_{i}-x_{i}}\right)},
$$

where $E_{i}$ is the $i$-th chaotic variable; $t_{i}$ is the sample size; $u_{i}$ is the predicted data; $k$ and $k+1$ represent the number of iterations.

The fuzzy layer makes the clear input of the input layer more fuzzy, and its connection weight with the upper layer neuron is roughly $i$, which is clearly changed to make it blur; the connection weight between this layer neuron and the upper part is $j$ when the membership function is calculated; the input and output relationship of this layer is

$$
R_{i j}=\sum_{i=1}^{n} \sum_{j=1}^{m}\left(\frac{o_{i j}+p_{i j}}{E_{i j}}-\frac{a_{i j}+s_{i j}}{E_{i j}}\right),
$$


where $R_{i j}$ is the center of the $j$-th membership function of the $i$-th input; $o_{i j}$ is the width of the $j$-th membership function of the $i$-th input; $a_{i j}$ is the number of variables of the $j$-th membership function of the $i$-th input; $s_{i j}$ is the learning rate of the $j$-th membership function of the $i$-th input.

2.2. Fuzzy Decision Algorithm. For chaotic time series $\left\{x_{1}, x_{2}\right.$, $\left.\cdots, x_{m}\right\}$, if a smaller value is assigned as $x_{0}$, the correlation function can be calculated after forming a phase space:

$$
T\left(x_{i}\right)=\frac{f\left(x_{i}\right)}{d x_{i}-h x_{i-1}}-\frac{g\left(x_{i}\right)}{d x_{i}+h x_{i-1}},
$$

where $T(i)$ is the distance between the vectors in the phase space; $f\left(x_{i}\right)$ is the radial basis function; $g\left(x_{i}\right)$ is a cumulative distribution function, representing the distance between two points on the space attractor; $d$ is the number of points in phase space; $h$ is the time point corresponding to the first local minimum.

Radial basis function fuzzy decision is a local approximation network, with a typical three-layer network structure; taking the number of inputs of the network as $l$ and the number of outputs as $z$, then the mathematical expression of the fuzzy decision completion mapping $f$ is

$$
Y\left(x_{i}\right)=\log \left[l \cdot U\left(x_{i}\right)-z \cdot I\left(x_{i}\right)\right],
$$

where $Y\left(x_{i}\right)$ is the input vector of the network; $l$ is the radial basis factor; $z$ is the norm; $U\left(x_{i}\right)$ is the connection weight of output layer; $I\left(x_{i}\right)$ is the center of the radial basis function.

For the $m$-dimensional phase space, if the phase point $x_{0}$ at the initial time $t_{0}$ is taken as the base point, the point $x_{i}$ closest to $x_{0}$ is selected from the remaining related points to form an initial vector; then the nearest point $x_{j}$ is selected until the trajectory under consideration is completed. With all those data, the maximum exponent $O_{i j}(x)$ can be obtained:

$$
O_{i j}(x)=\alpha \frac{e^{x_{i}}-e^{x_{j}}}{e^{x_{i}}+e^{x_{j}}}-\beta \frac{e^{x_{i}}}{e^{x_{j}}},
$$

where $\alpha$ is the number of evolutions on the reference trajectory; $\beta$ is the distance between the two trajectories after a period of evolution. Supposing the $A_{i j}$ group data $\left(x_{i}, x_{j}\right)$ belong to the sample set corresponding to node $x_{i} \in N(i=1,2$, $\cdots, n)$, the linear approximation function on node $x_{i}$ is defined as

$$
A_{i j}=\prod_{i, j=1}^{n} \frac{Y\left(x_{i}\right)-T\left(x_{i}\right)}{O_{i j}(x)} .
$$

The methods such as mutual information and autocorrelation function are used to delay the time of each subseries constituting a multivariable time series; according to the structural risk minimization principle, the optimization problem is transformed into finding object function $F$ $\left(x_{i}\right)$ :

$$
F\left(x_{i}\right)=\sqrt{\sum_{i=1}^{n}\left[\frac{x_{i}^{\mu}}{\tau(\gamma-1)}-\frac{x_{i}^{\sigma}}{\varphi(\rho-1)}\right]},
$$

where $\mu$ is the weight vector; $\gamma$ is the bias; $\sigma$ is the penalty coefficient; $\rho$ is the error; $x$ is the value of the input variable; $\tau$ is the corresponding output variable; $\varphi$ is the kernel parameter for automatic search.

\section{Economic Chaotic Time Series Prediction Model}

3.1. Parameter Synchronization Optimization. Under normal circumstances, it is easy to obtain a set of time series values in an economic system, that is, one-dimensional information of the system; this information contains all the characteristics of the system, but due to this onedimensional representation, the dynamic and multidimensional characteristics of the system can be reflected in some multidimensional information [9]. This method not only exerts the orbit prediction ability of the chaotic dynamics system due to the period density, but also avoids the influence of the inaccurate single prediction value caused by the sensitivity of the initial value, which can be used to predict the inflection point of the chaotic economic time series. In practice, due to the inability to obtain the complete time series of the chaotic system and the presence of noise interference, people cannot obtain complete system information from the time series and thus cannot restore the system space more accurately through reconstruction of the time series. This phase space reconstruction can only approximately replace the original chaotic space, and the prediction accuracy on it will continue to decrease as the prediction step size increases. The phase space structure in the longer time prediction step size should also change. In this way, the traditional algorithm for solving the embedded dimension and time delay is no longer suitable for the changed phase space structure, so a multistep prediction of economic chaotic time series is very important to find a more general theoretical basis.

The transfer of risk elements between projects has chaotic characteristics, and each project contains several risk elements. Therefore, chaotic series are used to initialize the risk status of each project in the project set. The item set risk element chaos initialization first initializes the item set with a chaotic series generated by the hybrid chaotic map; then, using the chaotic series of the initial item set as the initial value, a new chaotic series is generated by the hybrid chaotic system. Therefore, the project goals achieved by the probability model are very different from the actual situation. Although this completely random evolutionary process can ensure the continuous advancement of evolution as a whole, it is a slow and random process to eliminate a large number of unsolved solutions and continuously improve the fitness of each generation. This wide variety of poorly harvested algorithms is obviously of more computationally low efficiency. When the fitness value of an individual in a population of a certain generation is not much different from the 
global optimal solution of the fitness value function, the difference between individuals becomes small and the continuous reduction of the search area may cause the result to fall into the local optimum of solution domain and premature convergence. This phenomenon is especially likely to occur when a local optimal solution is close to the global optimal solution [10]. The analytical framework of economic chaotic time series prediction based on chaotic genetics combined with fuzzy decision algorithm is shown in Figure 1.

The essence of dynamic optimization scheduling is how to configure the schedulable time period of the supply chain member companies so that they can obtain the maximum output with the minimum supply chain cost. Regarding the form of the scheduling period, there are roughly two options for reference in existing studies: one is a continuous period; the scheduling period of each manufacturer cannot be divided, and the output is simply obtained based on the production time of a single piece [11]. Individuals selected by roulette are used to exchange some of their genes with a certain cross-probability to produce new gene combinations, which makes it possible for individuals to exchange their excellent genes and produce better solutions than their parents. In the later stage of the evolution, in order to avoid destroying the existing outstanding groups, the mutation probability is reduced, thereby improving the local finetuning function of the algorithm. In this paper, the twopoint crossover method is used to randomly select two crossover points for the selected parent individuals and exchange the servers corresponding to all tasks between the crossover points. The two-point crossover method can not only predict the time series of a chaotic system with fixed parameters, but also achieve accurate prediction when the chaotic system parameters are time-varying. Although chaos is random and unpredictable on the surface, this method actually moves according to strict and deterministic rules; that is, the movement should be predictable within a certain period of time.

3.2. Moderate Function Construction. The genetic algorithm simulates the biological evolution process in nature and finds the optimal solution of the problem with probability random search. The chaotic genetic algorithm generates a set of chaotic series through chaotic deterministic mapping, uses the carrier to convert the chaotic series with uniform distribution characteristics into optimization variables, and introduces the selection, crossover, and mutation operations of the genetic algorithm to optimize the fitness function value. Applying chaotic small disturbances to the optimal individuals selected by genetic operations effectively avoids premature convergence and obtains the global optimal solution. The buying and selling information of market actors is reflected in the price of the market. Only unforeseen events will affect the price changes of stocks, but the impact of random events on stock prices may be positive or negative, so the price change is the sum of the predictable trend part and the unpredictable random walk part. In fact, this is not the case in the market with little order, and the huge fluctuations in the stock market and the high degree of autocorrelation of time series all indicate the nonlinear characteristics of the market (Figure 2). Complexity theories such as fractal and chaos can quantify it, and an orderly process can be found from the results of complex and changeable price changes, so that the orderly nature of this process can be used to analyze and predict the more complicated economic chaotic time series [12].

For the observation time series from the deterministic chaotic system, short-term prediction can be made and radial basis function fuzzy decision is used for prediction. Radial basis function fuzzy decision is a kind of widely used forward nonfeedback fuzzy decision based on the function approximation theory. The basis function selected by the network has radial symmetry and is suitable for approximating arbitrary multivariate functions and their derivative values. The radial basis function fuzzy decision is composed of input layer, hidden layer, and output layer and the input layer node only transmits the input signal to the hidden layer [13]. The hidden layer node is composed of a radial action function like a Gaussian kernel function, and the output layer node is usually a simple linear function. In terms of the ability to generate new individuals in the process of genetic operation, crossover operation is the main method for generating new individuals. It determines the global search capability of genetic algorithm, while mutation operation is only an auxiliary method for generating new individuals, but it is also indispensable (Figure 3). It has one less operation step, because it determines the local search capability of the genetic algorithm. The chaotic optimization algorithm maps the optimization problem model to the chaotic variables and makes full use of the ergodicity, randomness, and regularity of the chaotic variables in the chaotic movement process to find the global optimal solution.

With the increase of fuzzy rules, the modeling accuracy of various methods has been improved, indicating that increasing the number of fuzzy rules helps improve the accuracy, but the corresponding modeling time also increases. In the traditional case, the error index is the root mean square error under the assumption of normal distribution and the information it contains is mainly accurate. Minimizing the root mean square can minimize the training error, but it will cause the fuzzy decision overlearning phenomenon, because the error in the distribution in practice may not be normal. For the control problem of stochastic system, it is always assumed that the random variable marrow obeys the normal distribution, so the control is concentrated on the performance indicators of mean and variance. However, in practice, the random variables of many systems are nonnormally distributed, and it is necessary to control the output probability density function or minimize the uncertainty of the system [14]. During the training process, the number of neurons is constantly changing, because the algorithm self-organizes one by one based on the training samples, so it can be seen that pruning and adding neurons are performed at the same time. The pruning algorithm based on the error entropy criterion and the error root mean square criterion can reasonably tailor the network structure. Under the premise 


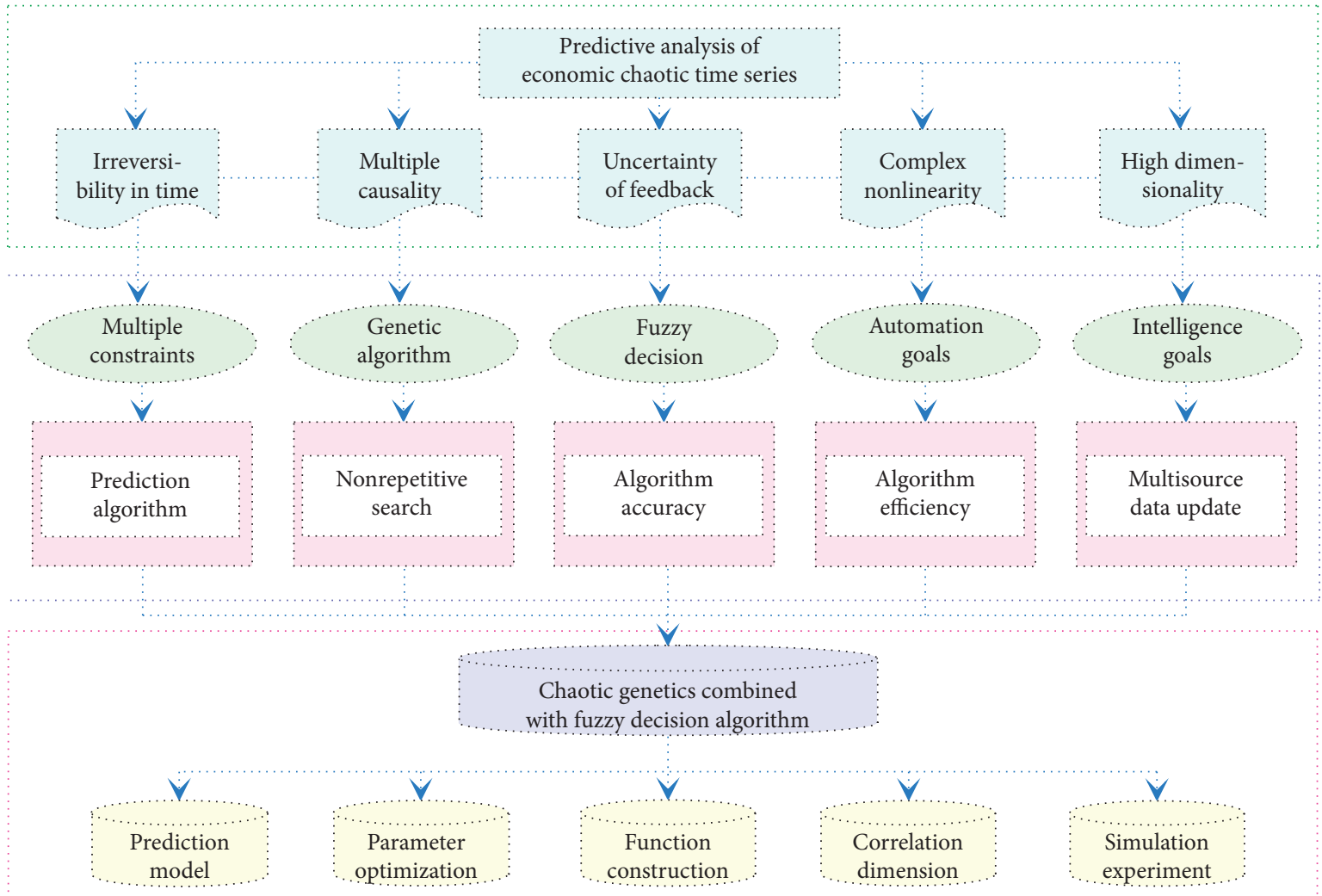

Figure 1: Analytical framework of economic chaotic time series prediction based on chaotic genetics combined with fuzzy decision algorithm.

of ensuring the training accuracy, maintain good generalization ability as much as possible, and make up for the overlearning in the traditional fuzzy decision algorithm disadvantages.

\section{Economic Chaotic Time Series Prediction Processes}

4.1. Phase Space Reconstruction. The basis of economic chaotic time series prediction is the theory of state space reconstruction, which believes that the evolution of any component of the system is determined by other components interacting with it, so it can be extracted and recovered from a batch of time series data of a certain component in the original law of the system. The kernel function is used to map the economic chaotic time series from the original space to the high-dimensional feature space into linear problem solving, which can bypass the specific form of the mapping, thereby effectively avoiding the dimensionality disaster problem. Fuzzy decision uses heuristic methods to solve specific problems, usually by trial and error, and largely depends on the experience of experimenters. Although fuzzy decision can achieve higher accuracy, it takes a lot of time to achieve the same accuracy index of finding out all kinds of information from it, and better understanding, mastering, and using its laws are undoubtedly of great significance to the prediction, decision-making, and risk management activities of import and export markets (Figure 4). The traditional time series analysis of total import and export volume is based on stochastic theory and is analyzed through regression methods. However, research shows that many economic time series often have deterministic and chaotic characteristics, so they are widely used in the analysis of economic time. The widely used economic chaotic time series method to analyze the time series of total imports and exports undoubtedly provides a new research method [15].

If the total supply chain cost of the production, storage, and transportation of two adjacent genes in the parent cluster can be found to be the largest among all adjacent genes in the parent cluster, then the two adjacent genes are probably not reasonable. The Lyapunov exponent calculation has radial symmetry and is suitable for approximating arbitrary multivariate functions and their derivative values, which can only approximately replace the original chaotic space, and the prediction accuracy on it will continue to decrease as the prediction step size increases [16]. If the new chromosome string after the swap has a higher fitness, the mutation operation is successful; otherwise, another random gene is replaced with it, trying to increase its fitness value (Figure 5). Through the dynamic scheduling time period model, it can be found that the problem is extremely complex, not only choosing the supply chain collaborator, but also choosing the call time of each partner; not only ensuring that the component manufacturer can call the time to meet the downstream assembly needs, but also ensuring 


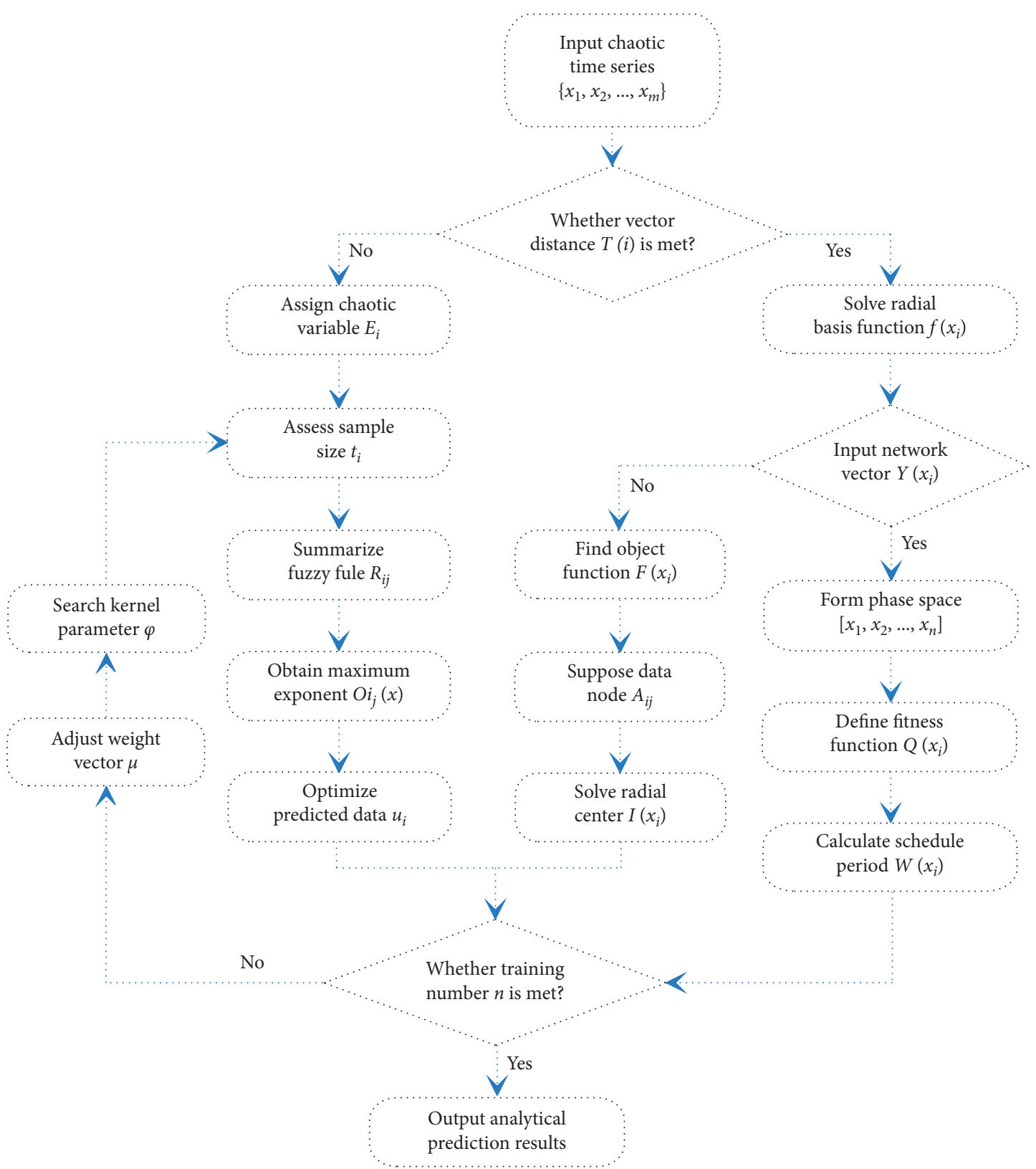

Figure 2: Analytical flowchart of economic chaotic time series prediction based on chaotic genetics combined with fuzzy decision algorithm.

that the component production suppliers obtain materials from upstream suppliers in time and start work normally; not only having to balance production capacity and demand planning, but also balancing storage and transportation capabilities; not only controlling production costs, but also controlling transportation and storage costs. Those with higher fitness values are more likely to be selected first, and their proportion in offspring will also increase, so that offspring will continue to outperform their parents.

During the evolution process, the evolution of any component in the system is determined by other components interacting with it, and the original law of the system can be extracted and restored from a batch of time series data of a certain component. Therefore, when performing phase space reconstruction, only one component needs to be investigated, and its measurement at certain fixed time delay points is treated as a new dimension, which determines a point in a certain multidimensional state space [17]. The crossover method adopts a partial matching crossover correction strategy. Four crossover points are selected on the four segments of the chromosome, and then a partial matching crossover strategy is used for another chromosome segment by segment. After the crossover is completed, the generated offspring is checked whether it is in the variable selection within the value range. Although the fuzzy decision obtained by the pruning method can structurally ensure 


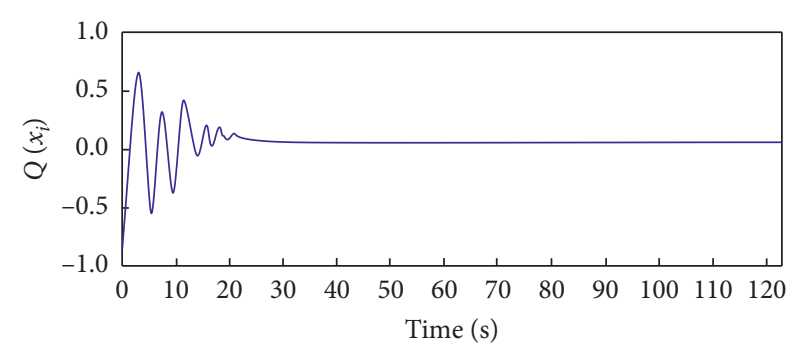

(a)

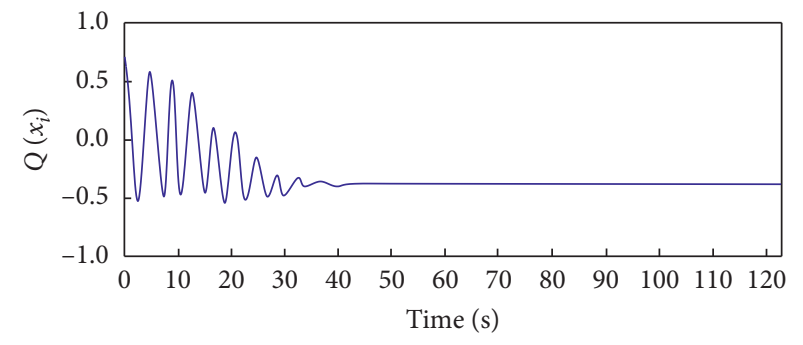

(c)

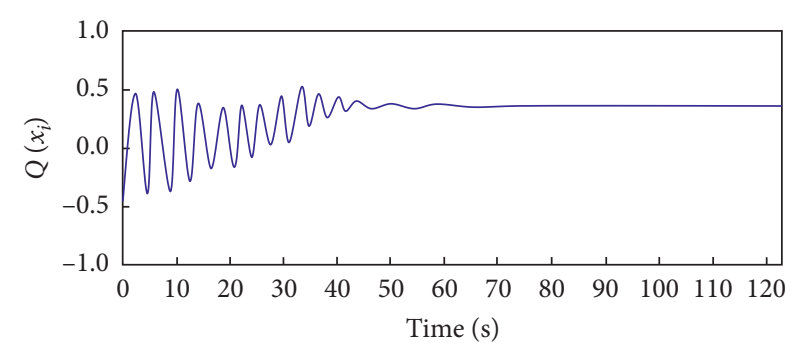

(b)

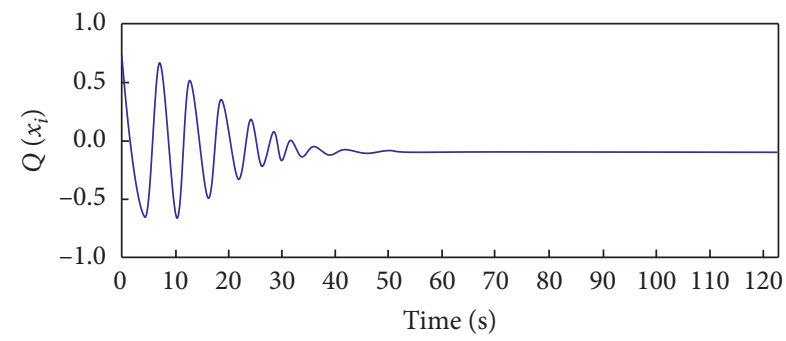

(d)

Figure 3: Relationship between total actual cost $\left[(\mathrm{Q})\left(x_{i}\right)\right]$ and delay time with four different corresponding output variables $(\tau)$.

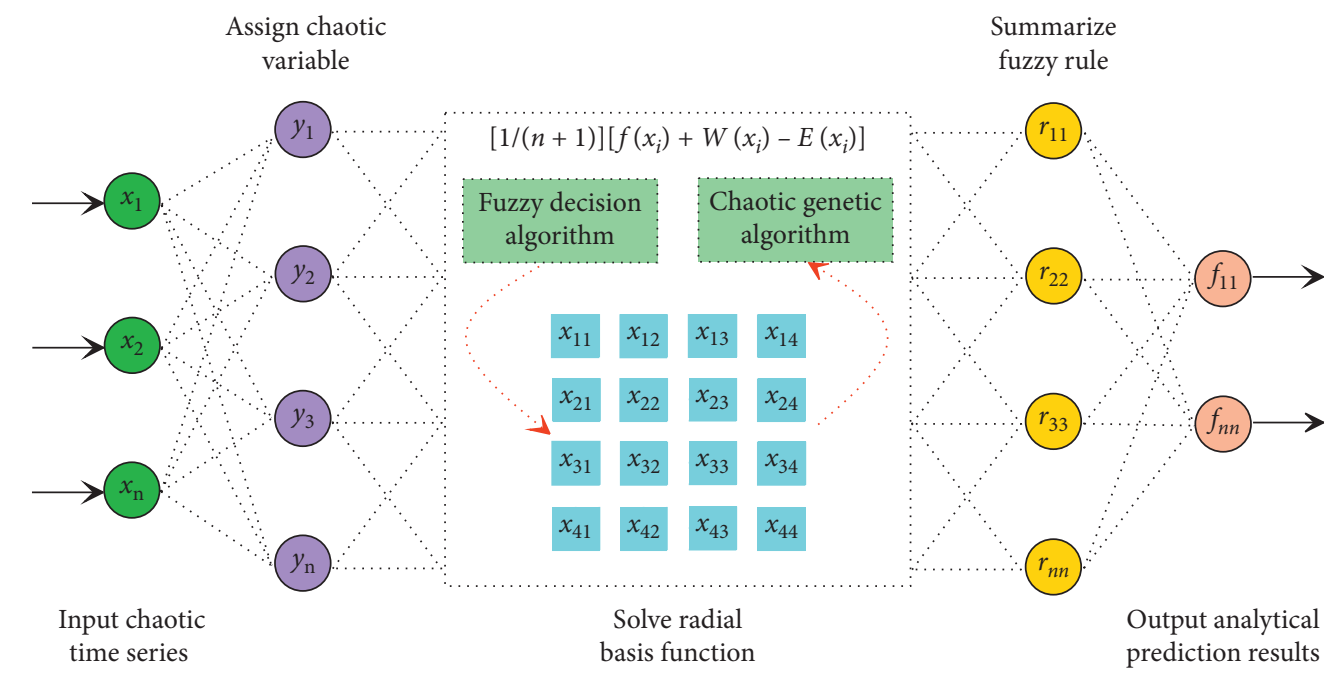

FIGURE 4: Economic chaotic time series prediction processes based on chaotic genetics combined with fuzzy decision algorithm.

that the network has a certain generalization performance, the final weights obtained by the pruning method are not optimal and need to be retrained. The genetic algorithm flexibly applies the biological evolutionary thoughts of nature to the field of optimization calculations. It can process several points in the space at the same time, thereby helping to search for the global optimal point, avoiding falling into the local minimum, and being especially effective when the error function is not differentiable or there is no gradient information at all. Lowdimensional chaotic systems can basically solve the network structure selection through phase space reconstruction theory, but it is not completely suitable for high-dimensional systems and data with a high degree of chaos is often encountered.
4.2. Correlation Dimension Calculation. On this basis, the system automatically selects assets to form an investment portfolio, measures and evaluates the performance of the investment portfolio, and timely feedbacks the investment situation to the investment manager. This is a dynamic cyclical process, which can ensure that the investment portfolio always obtains the maximum investment income at a satisfactory risk level, realizes the automation of the decision-making of investment portfolio management, and can provide users with dynamic portfolio management services. The Lyapunov exponent calculation is easy to obtain a set of time series values in an economic system, that is, one-dimensional information of the system; this information contains all the characteristics of the system [18]. Therefore, system 


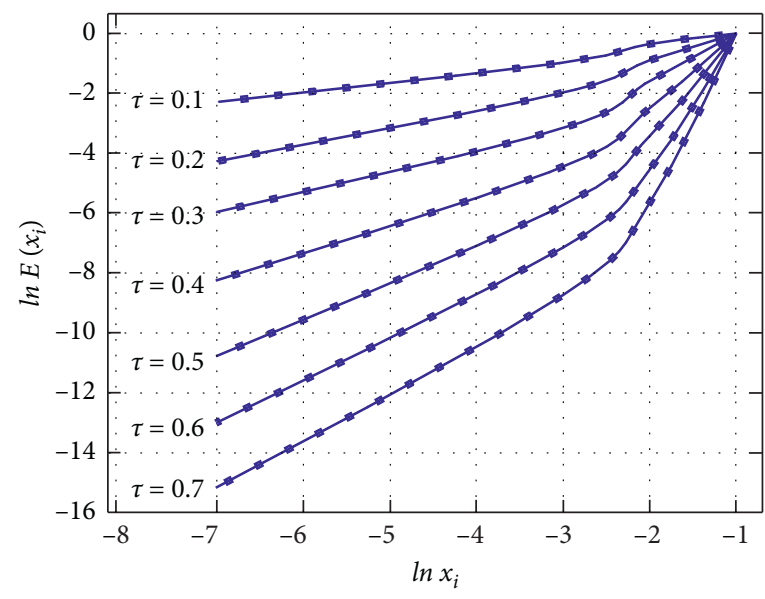

(a)

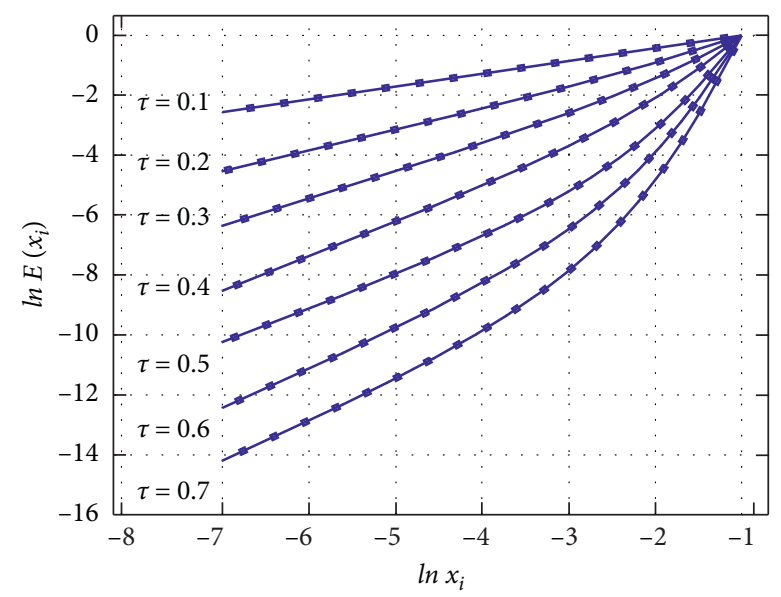

(b)

Figure 5: Relationship between $\ln E\left(x_{i}\right)$ and $\ln x_{i}$ when the variable is true value (a) and predicted value (b) with different corresponding output variables $(\tau)$.

prediction accuracy has always been the primary factor to consider when choosing a prediction model, and it is also the focus of system prediction theory research. Applying chaos theory to system prediction effectively improves the prediction accuracy and provides a powerful tool for system prediction. The phase space formed by the original state variables of the system is equivalent to the dynamic behavior in the reconstruction phase space of the onedimensional observations. The chaotic attractors in the two phase spaces are differential homeomorphisms; that is, the one-dimensional observations contain all the state variables of the system information about evolution. From this evolution law, the state of the system at the next moment can be obtained, and the predicted value of the next moment in the time series can be obtained, which provides a basis for the prediction of economic chaotic time series (Figure 6).

Although the autocorrelation function is a simple method to calculate the delay time, it can only extract the linear correlation between time series. The average displacement method belongs to the phase space reconstruction geometric method, which can be linked to the correlation criterion, but this method may contain strong jitter in the overall change trend and has a certain degree of randomness. The economic chaotic time series has a certain internal regularity, which is manifested as the correlation of the time series in the time delay state space. However, its nonlinear characteristics are difficult to directly find a function to describe the law of its development, and the autocorrelation method is one of the serial correlation methods. For high self-learning ability and the characteristics of being able to approximate nonlinear functions with arbitrary precision, fuzzy decision is an effective method. The evolution of any component of the system is determined by the other components interacting with it, and the relevant component information is implicit in the development of any component. Therefore, the sum can be extracted from the time series data of a certain component and restored from the original rules of the system. This method first uses the genetic algorithm to perform a global search in the solution space and then uses the fuzzy decision learning to find the optimal solution in the optimal interval searched by the genetic algorithm, which has a better nonlinear fitting ability for economic chaotic time series and higher prediction accuracy.

The position and structure of each block in the chromosome are relatively fixed, so that the design of crossover and mutation operations can be greatly simplified. The number of blocks and the number of units in each block can be expanded according to this growth law, so as to obtain more complex function expressions. The Lyapunov exponent calculation is merged into one rule with the most occurrences being regarded as the rule after the merger, and its confidence is regarded as the confidence of the rule, and the number of occurrences is the sum of the occurrences of the same rule of all the antecedents. The marked standard deviation curve is the standard deviation corresponding to the fitness function value of the entire population, and local modeling depends on the specific information of a certain part of the attractor. There are zero-order, first-order, second-order, and high-order local models and nonlinear fuzzy decision local models; a new set of solutions is generated by simulated evolution and inherited genetic operations, and each solution is evaluated by an objective function. This process is repeated until a certain form of convergence is reached; a new set of solutions can not only selectively retain some old solutions with high objective function values, but also include some new solutions obtained by combining other solutions.

\section{Simulation Experiment and Analysis}

5.1. Experimental Design. The steps for the simulation experiment of economic chaotic time series are as follows: first, 1000 sample data are used to build a fuzzy rule library to 
build the entire fuzzy decision structure; second, the network is trained to adjust the parameters of the membership function to make the entire fuzzy decision system performance when the indicator reaches the expected value; third, the learning of the original fuzzy rules is optimized to use the optimized model to predict the future value when there are new data pairs; fourth, the training data are divided into two parts with one part used for the training of each subnetwork and the other part used for the verification of the entire model; fifth, the division point is coded and the genetic algorithm is used to find the optimal division point; that is, the initial division point divides the reconstructed phase space point into several subspaces-if the reconstructed phase space has $n$ dimensions, the initial division point will divide the phase space into $2 n$ subspaces, and each point in the subspace corresponds to a subnetwork module for training; sixth, when all subnetwork modules are trained, the error of the division point on the verification data is obtained-after the error is sorted, the last $25 \%$ of the division points with the larger error are eliminated; then a new randomly generated division point is added to enter the next generation until the prediction is obtained.

Predictive analysis of economic chaotic time series is a dynamic cyclical process, in which the model can be predicted by ensuring that the investment portfolio always obtains the maximum investment income at a satisfactory risk level, realizes the automation of the decision-making of investment portfolio management, and can provide users with dynamic portfolio management services. Based on the idea of data mining, the redundant and conflicting initial fuzzy rules are pruned to reduce the large amount of redundant information generated in the identification process. The confidence measure is defined to reflect the reliability of the generated rules, which provides a basis for further rule pruning and analysis and an improved gradient descent method. Not only can the fuzzy model be identified at the same time, its parameters can be adjusted and the optimal output fuzzy subset can be determined, but the accuracy of the fuzzy predictor can also be greatly improved. By introducing a dynamic error transfer factor, the conflict between convergence speed and oscillation in the gradient descent method is solved. The main idea is that the evolution of any component of the system is determined by other components interacting with it, and the information of these related components is implicit in the development of any component. Therefore, the original law of the system can be extracted and restored from a batch of time series data of a certain component, which is a trajectory in a high-dimensional space.

5.2. Result Analysis. Practical research shows that a new prediction method that combines the least squares method suitable for solving large-scale problems and the vector machine theory based on fuzzy models has unique advantages in the modeling of complex nonlinear systems. Since economic chaotic time series are extremely sensitive to the initial state, it brings great difficulties to the prediction of economic chaotic time series. However, experiments show that the prediction of economic chaotic time series is not only feasible, but also accurate within a certain range using vector machines based on fuzzy models. Usually the prediction effect of the model is related to the delay time and embedding dimensions. If the time is too small, the difference between the adjacent delay coordinate elements of the input vector is too small; that is, the redundancy is large, and the information about the original attractor contained in the reconstructed phase space sample point is too small, which is reflected in the phase space form above, and the signal trajectory is compressed toward the main axis of the phase space (Figure 7). On the contrary, if the dimension is too large, the mutual information between the delayed coordinate elements in the phase space is lost; that is, the elements are not correlated, and the signal trajectory may be folded. In addition, for different embedding dimensions, the optimal delay time is also different. Therefore, when building a model of economic chaotic time series, it is necessary to choose an appropriate delay time.

When this method is faced with some time-varying chaotic series with long periodicity, it is difficult to select the most relevant historical samples into the training set with a short window length, thus affecting the effect of machine learning. If a long window is used for sample selection, the machine learning time is longer and it is not suitable for some occasions with high timeliness requirements. According to the infinitely nested self-similarity of economic chaotic time series, the development trajectory of this sample point and its nearest neighbor is similar. Therefore, the nearest neighbor contains the largest prediction-related information predicted. Similarly, the sample point set with the smallest distance to the prediction sample is located on the nearest neighbor orbit set to the prediction sample, and it also has a great correlation with the prediction sample (Figure 8). So it can be said that the prediction sample in the economic chaotic time series is related to the distance between the training samples. Therefore, it can not only predict the time series of a chaotic system with fixed parameters, but also achieve accurate prediction when the chaotic system parameters are time-varying. The sample subset obtained in each update includes both time-dependent samples and the samples most similar to the predicted samples, which is suitable for processing some long-period timevarying chaotic series.

When the dimensionality of the input variables of the fuzzy logic system is higher and each variable has more fuzzy partitions, the number of fuzzy rules increases exponentially and becomes very large. Although there are many rules that describe a system, their roles are different and some fuzzy rules do not appear at all or have a small probability of appearing. These rules are redundant and can be removed from the rule base to simplify the network and speed up calculations. Using this system, the mapping from input to output can be obtained, and its accuracy depends on the accuracy of the membership function and the rule base. When the reasoning accuracy 


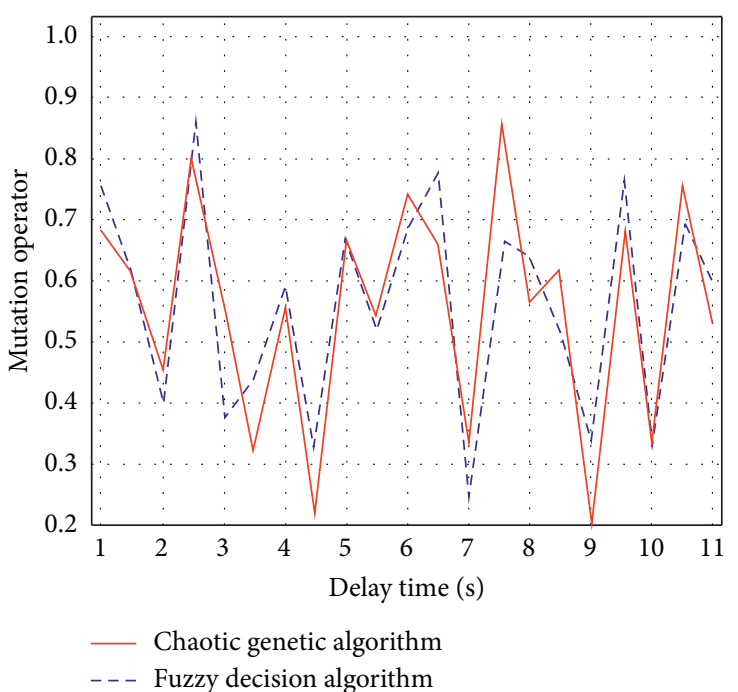

(a)

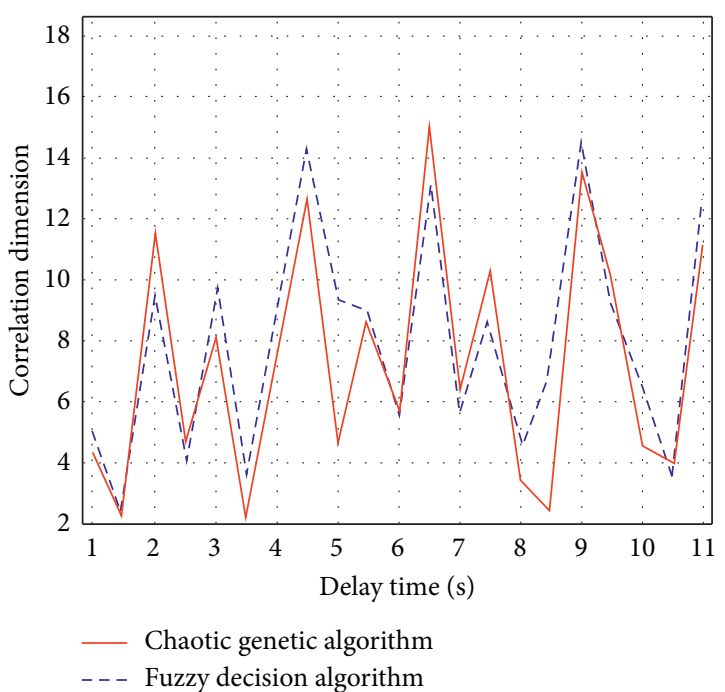

(b)

FIgURE 6: Relationship between delay time with mutation operator (a) and correlation dimension (b) of chaotic genetic and fuzzy decision algorithm.

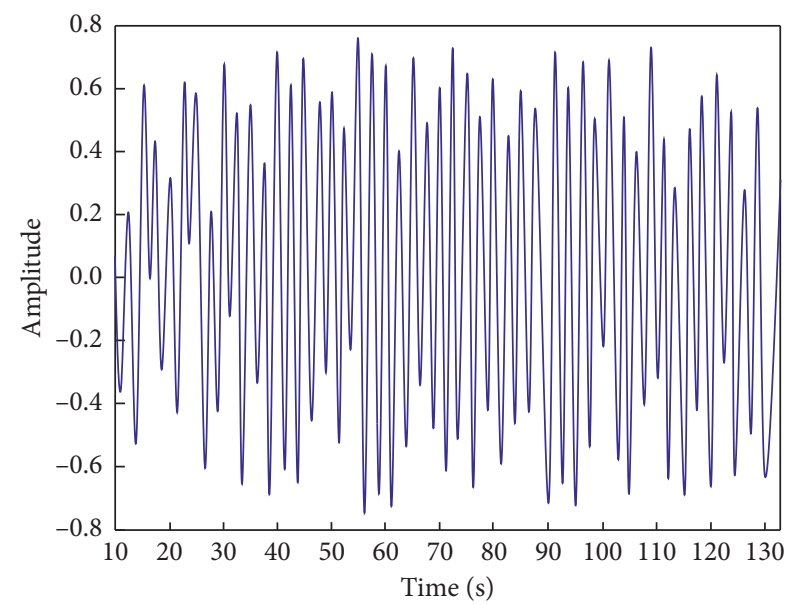

(a)

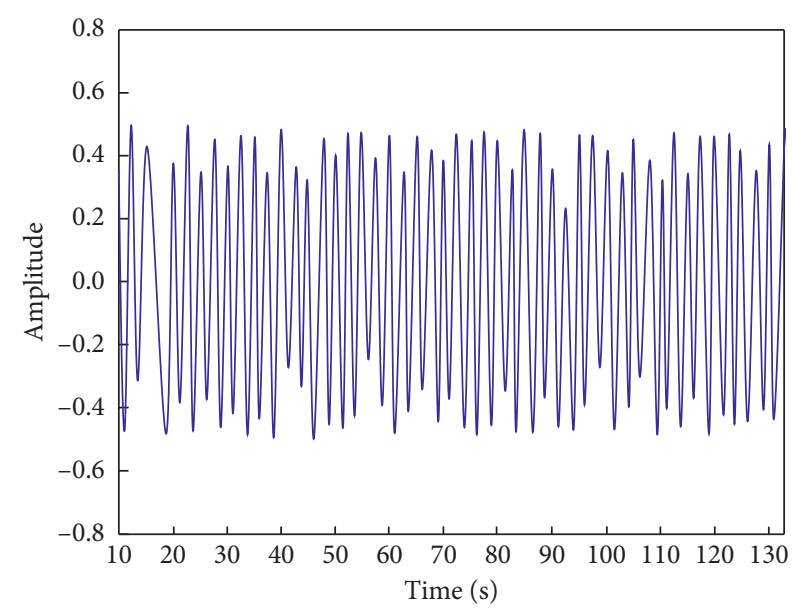

(b)

FIGURE 7: Economic chaotic time series based on chaotic genetics combined with fuzzy decision algorithm when the variable is true value (a) and predicted value (b).

of the system is not high enough, the parameters of the membership function and the weight of fuzzy decision need to be adjusted and optimized. The rules with the same antecedent and different subsequent ones are merged into one rule, the rule with the most occurrences is regarded as the rule after the merger, and its confidence is regarded as the confidence of the rule, and the number of occurrences is the sum of the occurrences of the same rule of all the antecedents. In the fuzzy decision, the learning process is divided into two parts: the first part is the structure learning phase, which processes the input data, extracts the best fuzzy rules, and determines the structure of the entire system; the second part is the parameters in the learning stage; the backpropagation algorithm is used to adjust the network weights and membership function parameters. 


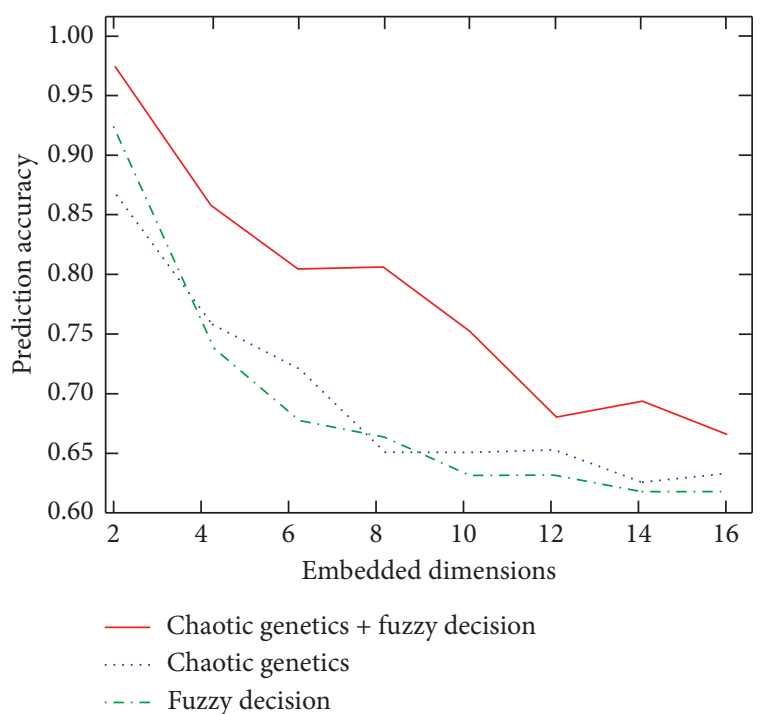

(a)

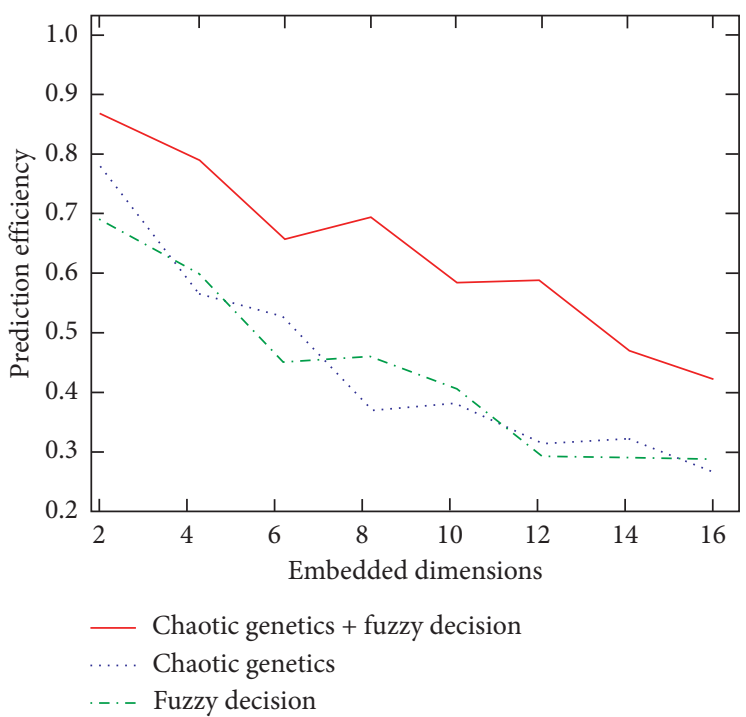

(b)

Figure 8: Relationship between embedded dimensions with prediction accuracy (a) and efficiency (b) with the three different algorithms, respectively.

\section{Conclusions}

This paper constructed a prediction model for economic chaotic time series, performed parameter synchronization optimization and moderate function construction, analyzed the prediction processes of economic chaotic time series, conducted phase space reconstruction and correlation dimension calculation, and finally carried out a simulation experiment with its result analysis. The economic chaotic time series has a certain internal regularity, which is manifested as the correlation of the time series in the time delay state space. The marked standard deviation curve is the standard deviation corresponding to the fitness function value of the entire population, and local modeling depends on the specific information of a certain part of the attractor. High self-learning ability enables approximate nonlinear functions with arbitrary precision; therefore, fuzzy decision is an effective method. With the increase of fuzzy rules, the modeling accuracy of various methods has been improved, indicating that increasing the number of fuzzy rules helps to improve the accuracy, but the corresponding modeling time also increases. Although the fuzzy decision obtained by the pruning method can structurally ensure that the network has a certain generalization performance, the final weights obtained by the pruning method are not optimal and need to be retrained. A new set of solutions is generated by simulated evolution and inherited genetic operations, and each solution is evaluated by an objective function. The study results show that the algorithm of chaotic genetics combined with fuzzy decisionmaking can dynamically adjust chaotic mutation operators and summarize fussy expert experiences. The phase space of its reconstructed chaotic attractor has high-precision predictability and can find orderly processes from changeable economic results, which in turn can be used to analyze and predict the complex economic chaotic time series. The study results of this paper provide a reference for further research on predictive analysis of economic chaotic time series based on chaotic genetics combined with fuzzy decision algorithm.

\section{Data Availability}

No datasets were generated or analyzed during the current study.

\section{Conflicts of Interest}

The authors declare that they have no conflicts of interest.

\section{Authors' Contributions}

All authors approved the publication of the paper.

\section{Acknowledgments}

The authors acknowledge the support from the Scientific Research Project of China (Xi'an) Institute for Silk Road Research: Study on Spillover Effect and Mechanism of Shaanxi Free Trade Zone on Cultural Trade (Project no. 2019YA04).

\section{References}

[1] B. R. Adarsh, T. Raghunathan, T. Jayabarathi, and X.-S. Yang, "Economic dispatch using chaotic bat algorithm," Energy, vol. 96, pp. 666-675, 2016.

[2] S. S. Askar, A. A. Karawia, and F. S. Alammar, "Cryptographic algorithm based on pixel shuffling and dynamical chaotic economic map," Iet Image Processing, vol. 12, no. 1, pp. 158-167, 2018.

[3] D. C. Secui, "A new modified artificial bee colony algorithm for the economic dispatch problem," Energy Conversion and Management, vol. 89, pp. 43-62, 2015. 
[4] H. Baskonus, T. Mekkaoui, Z. Hammouch, and H. Bulut, "Active control of a chaotic fractional order economic system," Entropy, vol. 17, no. 12, pp. 5771-5783, 2015.

[5] C. Luo, N. Zhang, and X. Wang, "Time series prediction based on intuitionistic fuzzy cognitive map," Soft Computing, vol. 24, no. 9, pp. 6835-6850, 2020.

[6] E. A. Bakeshlou, A. A. Khamseh, M. A. G. Asl, J. Sadeghi, and M. Abbaszadeh, "Evaluating a green supplier selection problem using a hybrid MODM algorithm," Journal of Intelligent Manufacturing, vol. 28, no. 4, pp. 913-927, 2017.

[7] H. Rezaie, M. H. Kazemi-Rahbar, B. Vahidi, and H. Rastegar, "Solution of combined economic and emission dispatch problem using a novel chaotic improved harmony search algorithm," Journal of Computational Design and Engineering, vol. 6, no. 3, pp. 447-467, 2019.

[8] M. Balasubbareddy, "Multi-objective optimization in the presence of ramp-rate limits using non-dominated sorting hybrid fruit fly algorithm," Ain Shams Engineering Journal, vol. 7, no. 2, pp. 895-905, 2016.

[9] A. Rajagopalan, V. Sengoden, and R. Govindasamy, "Solving economic load dispatch problems using chaotic self-adaptive differential harmony search algorithm," International Transactions on Electrical Energy Systems, vol. 25, no. 5, pp. 845-858, 2015.

[10] A. Man-Im, W. Ongsakul, J. G. Singh, and C. Boonchuay, "multi-objective economic dispatch considering wind power penetration using stochastic weight trade-off chaotic NSPSO," Electric Power Components and Systems, vol. 45, no. 14, pp. 1525-1542, 2017.

[11] S. Khan, J. Ahmad, I. Naseem, and M. Moinuddin, "A novel fractional gradient-based learning algorithm for recurrent neural networks," Circuits, Systems, and Signal Processing, vol. 37, no. 2, pp. 593-612, 2018.

[12] M. Balasubbareddya, S. Sivanagarajub, C. Venkata Sureshc, A. V. Naresh Babud, and D. Srilathaa, "A non-dominated sorting hybrid cuckoo search algorithm for multi-objective optimization in the presence of FACTS devices," Russian Electrical Engineering, vol. 88, no. 1, pp. 44-53, 2017.

[13] W. Guo, T. Xu, and Z. Lu, "An integrated chaotic time series prediction model based on efficient extreme learning machine and differential evolution," Neural Computing and Applications, vol. 27, no. 4, pp. 883-898, 2016.

[14] S. Zhou and X. Wang, "Simple estimation method for the largest Lyapunov exponent of continuous fractional-order differential equations," Physica A: Statistical Mechanics and Its Applications, vol. 563, Article ID 125478, 2021.

[15] J. A. Bossard, L. Lin, and D. H. Werner, "Evolving random fractal cantor superlattices for the infrared using a genetic algorithm," Journal of the Royal Society Interface, vol. 13, no. 114, Article ID 20150975, 2016.

[16] M. Zhang, B. Wang, Y. Zhou, and H. Sun, "WOA-based echo state network for chaotic time series prediction," Journal of the Korean Physical Society, vol. 76, no. 5, pp. 384-391, 2020.

[17] H. Meng, C. Wang, A. Zhang, and J. Bao, "A novel chaotic time series prediction method and its application to carrier vibration interference attitude prediction of stabilized platform," Journal of Vibroengineering, vol. 18, no. 1, pp. 562-576, 2016.

[18] A. Tharwat and A. E. Hassanien, "Chaotic antlion algorithm for parameter optimization of support vector machine," Applied Intelligence, vol. 48, no. 3, pp. 670-686, 2018. 